EPJ Web of Conferences 31, 00039 (2012)

DOI: $10.1051 /$ epjconf/20123100039

(C) Owned by the authors, published by EDP Sciences - SIF, 2012

\title{
Decay of excited medium-mass compound nuclei
}

\author{
S. BARLini for the NUCLEX and HECTOR Collaborations
}

INFN and Dipartimento di Fisica dell'Universita, Firenze, Italy.

\begin{abstract}
An experimental campaign has been performed by the NuclexHector collaboration at the Laboratori Nazionali di Legnaro in order to study different reaction channels by detecting, in coincidence, Evaporation Residue (ER), Charged Particles and high energy $\gamma$-rays from Giant Dipole Resonance (GDR). The studied system was ${ }^{48} \mathrm{Ti}+{ }^{40} \mathrm{Ca}$ at 300, 450 and $600 \mathrm{MeV}$, using a modular apparatus formed by triplephoswich detectors for ER and particles at the forward angles, the GARFIELD $\triangle \mathrm{E}$-E forward drift chamber for the charged particles between $30^{\circ}$ and $85^{\circ}$ and the HECTOR apparatus at the backward angles for $\gamma$-rays.
\end{abstract}

Hot rotating nuclei in the region $A=100$ formed via fusion reactions have different de-excitation modes, from nucleon evaporation to fission and GDR emission. The study of this modes is interesting for the knowledge of the relaxation of both intrinsic and collective degrees of freedom, opening also new possibilities in view of future experiments with exotic beams. The present work was done with the aim to investigate in a rather complete and exclusive way the several decay processes of the ${ }^{88}$ Mo Compound Nucleus (CN), formed in fusion reactions between ${ }^{48} \mathrm{Ti}$ and ${ }^{40} \mathrm{Ca}$ at 300,450 and $600 \mathrm{MeV}$. A large part of the reaction cross section for these reactions is exhausted by fusion. At these three energies, for full momentum transfer, we produce CN with an excitation energy of 126, 194 and $262 \mathrm{MeV}$ respectively and with spin arriving at the critical values for fission $(1 \sim 65-70 \hbar)$. Decay of ${ }^{88} \mathrm{Mo}$ can occur via particle evaporation and fission (symmetric or asymmetric).

This is an Open Access article distributed under the terms of the Creative Commons Attribution License 2.0, which permits unrestricted use, distribution, and reproduction in any medium, provided the original work is properly cited. 

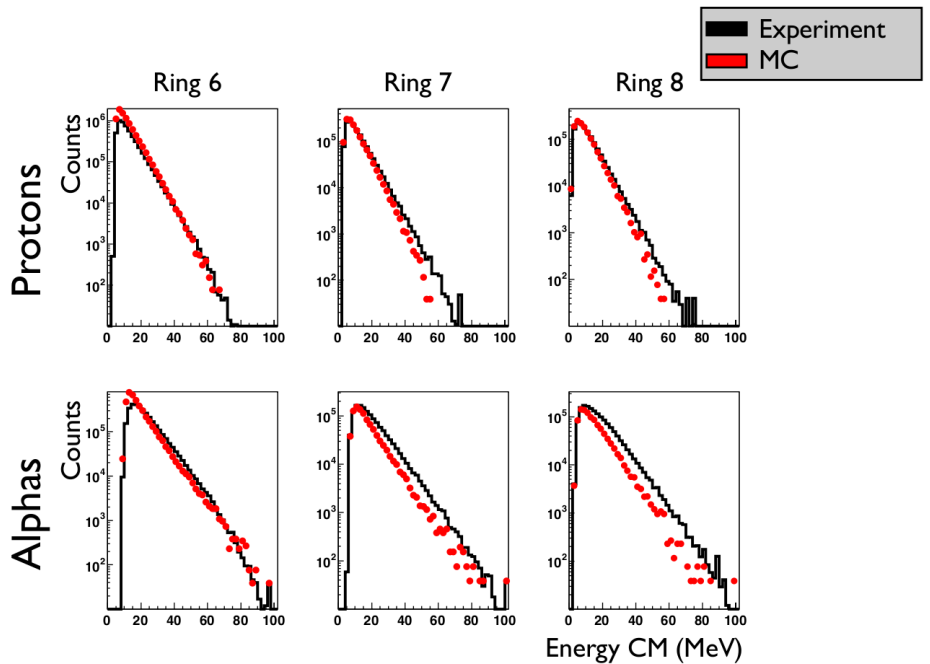

Figure 1: (Color Online) LCP spectra in coincidence with ER for different ring of the GARFIELD apparatus for the ${ }^{48} \mathrm{Ti}+{ }^{40} \mathrm{Ca}$ at 600 reaction.

Of course, other reaction modes besides fusion, like deep-inelastic reaction of quasi-fission processes can be present. A severe experimental task is just to separate these different mechanisms and decay modes in order to study their relevance, features and interplay ( [1], [2]).

The experiments analyzed in this work were done at Laboratori Nazionali di Legnaro (INFN) with a modular apparatus largely equipped with modern electronics based on digital sampling ADC and real-time DSP processing. The array was divided in three parts: a set of 50 triple-phoswich detectors [3] was placed far from the target (85 and $160 \mathrm{~cm}$ distance) at the forward angles to detect mainly ER, Fission Fragments (FF) and also charged particles. These phoswiches are composed of very fast plastic (0.2 mm thickness), a fast plastic $(5 \mathrm{~mm})$ and finally a $\mathrm{CsI}(\mathrm{Tl})(4 \mathrm{~cm})$. They are arranged in an azimuthally symmetric wall of 32 detectors between $6^{\circ}$ and $13^{\circ}$ and a set of 16 detectors around $20^{\circ}$ on both sides of the beam axis mainly to detect symmetric FF. The GARFIELD forward gas-CsI drif chamber [4] was used to complete charged fragment detection between $30^{\circ}$ and $85^{\circ}$, while the energetic gammas were measured by the 8 big $\mathrm{BaF}_{2}$ scintillators of the HECTOR group [5]. Here we report on an analysis only based on charged products to identify the fusion-evaporation or the fusionfission processes. The signals from the phoswich photomultiplier anodes are 


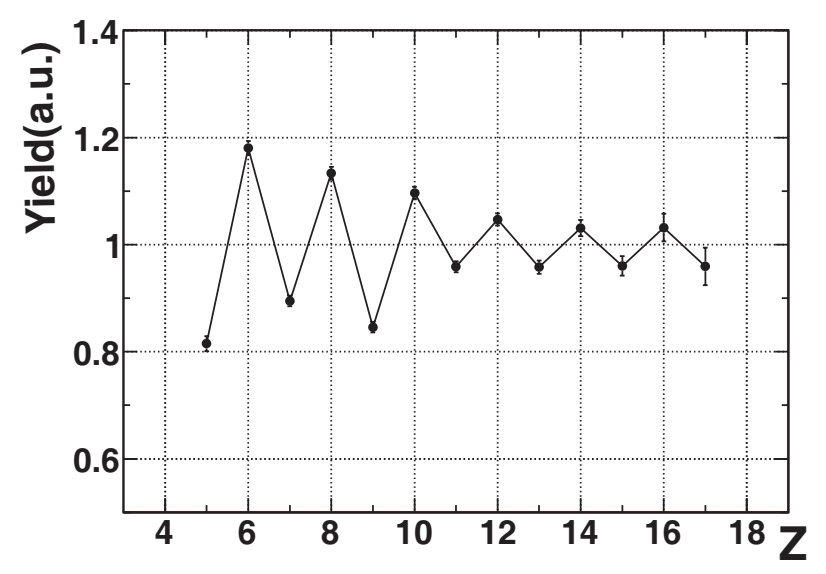

Figure 2: Charge distribution of the lighter FF, normalized using the technique discussed in [8] to enhanced the staggering effects.

sampled and digitized using a $125 \mathrm{MHz}$ card [6], developped in Florence. On these sampled signals several operations and analysis can be done. Namely, a digital constant fraction time mark $\left(\mathrm{t}_{c f d}\right)$ and three differents gates, gA, $\mathrm{gB}$ and $\mathrm{gC}$ are defined in order to perform a Time of Flight (ToF) measurement and disentangle the three different contributions coming out from the different scintillation layers. Using these parameters, different identification plots can be built. In particular, the ER selection is performed using a grafical cut on the gA-ToF plot. In Fig.1, the LCP energy spectra in the center of mass detected in coincidence with ER by different rings of the GARFIELD apparatus are shown for the $600 \mathrm{MeV}$ reaction. In particular, the cs6 ring corresponds to $53<\theta<66^{\circ}$, the $\operatorname{cs} 7$ to $41<\theta<52^{\circ}$ and $\operatorname{cs} 8$ to $29.5<\theta<40^{\circ}$. They are compared with the distribution obtained with the GEMINI code [7], using standard parameters, assuming complete fusion and a triangular spin distribution for the $\mathrm{CN}$ up to vanishing barrier values.

Using stringent cuts, it is possible to select events which are compatible with the fission of the fused ${ }^{88}$ Mo nuclei. These conditions consist in a double coincidence within the detectors, with the two fragments velocities back-to-back in the center of mass. In the case of asymmetric split, the lighter fragment can be identified in charge both in the Phoswich or in the GARFIELD apparatus up to $\mathrm{Z} \sim 12$. Recently, renewed interest in odd-even oscillations in the fragment yields or in other N/Z observables arose, due to the interest in studying basic properties of nuclei (level density, parity, 
shell-closure) at the interface between the single-state energy region and the continuum( [1], [8], [9]). Fig.2 presents preliminary results on staggering effects for the lighter FF in fusion-fission reactions at $600 \mathrm{MeV}$. The staggering is here presented following the zooming procedure suggested by D'Agostino et al. [8]. The present result looks like similar to that of Fig.6 from [8], where the fragments from the central collisions of the reaction ${ }^{32} \mathrm{~S}+{ }^{58-64} \mathrm{Ni}$ at $14.5 \mathrm{Mev} / \mathrm{u}$ has been studied. These two considered systems are prepared via different reactions, but with similar final energies and sizes.

\section{Conclusion}

The decay of hot rotating ${ }^{88} \mathrm{Mo}$ nuclei produced in the ${ }^{48} \mathrm{Ti}+{ }^{40} \mathrm{Ca}$ reaction at $600 \mathrm{MeV}$ has been studied. The evaporation LCP spectra are in reasonable agreement with the GEMINI statistical code (Fig.1), while the charge distribution of the lighter fragments of the fission process shows a staggering behaviour (Fig.2). Other observables will be investigated in the next future (as the fusion cross section, the LCP yield ratios) to better characterize the decay of n-poor CN in the evaporation channel. Moreover, the comparison of this reaction with the 450 and $300 \mathrm{MeV}$ reactions will help in undestanding the evolution of the different mechanisms as a function of the excitation energy.

\section{References}

[1] G.Ademard et al., Phys. Rev. C 83, 054619 (2011)

[2] K.X.Jing et al.,Nucl. Phys. A645, 203 (1999)

[3] M.Bini et al., Nucl. Inst. and Meth. A515, 497 (2003)

[4] F.Gramegna et al., Nucl. Inst. and Meth. A389, 474 (1997)

[5] O.Wieland et al., Phys. Rev. Lett. 97,012501 (2006)

[6] G.Pasquali et al., Nucl. Inst. and Meth. A570, 126 (2007)

[7] R.J.Charity et al., Phys. Rev. C 82, 014610(2010)

[8] M.D'Agostino et al., Nucl. Phys. A 861, 47(2011) and 875, 139(2012)

[9] I.Lombardo et al., Phys. Rev. C 84, 024613(2011) 